\title{
A low-power and high-precision miniaturized digital sun sensor
}

\author{
B.M. de Boer*, M. Durkut \\ TNO, stieltjesweg 1, 2600AD Delft, the Netherlands
}

\begin{abstract}
A prototype miniaturized digital sun sensor (miniDSS) was developed by TNO. It is expected to be launched on QuadSat for in-orbit demonstration. The single-chip sun sensor comprises an application specific integrated circuit (ASIC) on which an active pixel sensor (APS), read-out and processing circuitry as well as communication circuitry are combined. The sun sensor consumes only $65 \mathrm{~mW}$, has a volume of $69 \times 52 \times 14 \mathrm{~mm}^{3}$ and a mass of just 72 grams. Although the miniDSS is a miniaturized and low-power device, the accuracy is not compromised by this. The uncalibrated accuracy is in the order of a few hundreds of a degree, across the field of view of $102^{\circ} \times 102^{\circ}$. The sensor is albedo insensitive.
\end{abstract}

Keywords: Sun sensor, DSS, Active Pixel Sensor, AOCS sensor

\section{INTRODUCTION}

A prototype of a low-power and high-precision miniaturized digital sun sensor (miniDSS) was developed at TNO. Conventionally, high-precision sun sensors are large, expensive devices that moreover consume a relatively large amount of power ${ }^{1,2}$. The miniDSS, which is shown in Figure 1, provides a cost-effective, miniaturized and low-power alternative, with comparably high accuracy.

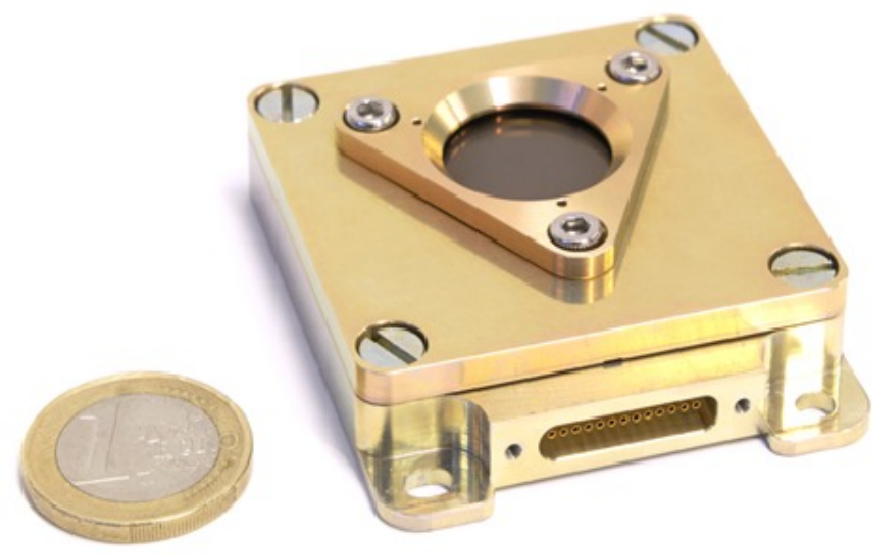

Figure 1: Photograph of the miniDSS prototype.

The fully-functional miniDSS prototype is expected to be launched on QuadSat in 2013 for in-orbit demonstration. This satellite program is a cooperation between AAC Microtec (Sweden) and OHB (Germany) and is designed to demonstrate Space Plug and Play Avionics (SPA) interface technology. Further development of the miniDSS towards qualified models is planned in close cooperation with Moog Bradford (formerly known as Bradford Engineering). Moog Bradford has collaborated with TNO on many occasions and currently produces large quantities of analogue sun sensors based on TNO designs.

* bart.deboer@tno.nl; phone +31 888660903; tno.nl 


\section{OPERATION}

The miniDSS comprises a custom-developed advanced active pixel sensor (APS) that is located behind a pinhole-sized aperture through which sun light penetrates and incidences on the pixel array. The principle of operation, which is shown in Figure 2, is based on accurate measurement of the location of the center of luminosity of the sun spot projected onto the APS. The location of the center of the sun spot is provided to the space craft (S/C) in the form of an $X$ and $Y$ value from which the $\mathrm{S} / \mathrm{C}$ can calculate sun aspect angles $\alpha$ and $\beta$. The relation between $Y$ and sun aspect angle $\alpha$ is given by

$$
\alpha=\arctan \left(Y \cdot \frac{s}{h}\right)
$$

in which $Y$ is the distance in pixels along the $y$-axis between the center of the APS and the center of the sun spot, $s$ is the pixel size and $h$ is the collimator height, i.e. the distance between the aperture and the APS planes. Similarly, sun aspect angle $\beta$ is calculated as

$$
\beta=\arctan \left(X \cdot \frac{s}{h}\right) \text {, }
$$

in which $X$ is the distance in pixels along the $x$-axis between the center of the APS and the center of the sun spot.

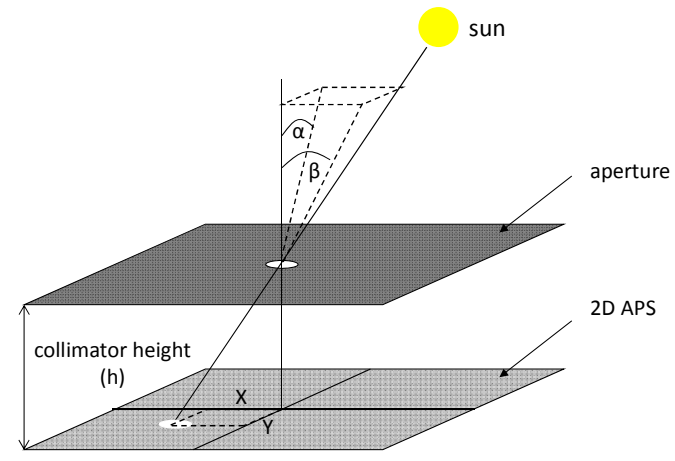

Figure 2: Operating principle of the miniDSS.

The S/C does not need to use a calibration look-up table (LUT) or perform temperature compensation to obtain highaccuracy sun aspect angles measurements with the miniDSS. The largest contribution to inaccuracy can be attributed to aperture misalignment. Small unit-specific deviations from the desired aperture position in the $x$-, $y$-and $z$-direction, with respect to the pixel array, are unavoidable as result of the placement tolerances during assembly. However, these misalignments are easily obtained from a very limited calibration procedure. In order to achieve a high accuracy, these misalignments can be compensated for by adding the misalignment values to $X, Y$ and $h$, respectively, when calculating the sun aspect angles. Without taking aperture misalignment into account the accuracy of the miniDSS prototype was found to be in the order of $0.3^{\circ}$ across the field of view (FOV), which may be sufficient for many applications. In the cases where such an accuracy suffices, a unit level calibration may be omitted, which significantly reduces the recurrent cost of the sensor. 
The miniDSS has two operating modes: An acquisition mode and a tracking mode. After power-up the miniDSS starts in the acquisition mode, in which the presence and coarse location of the sun spot on the pixel array are determined. When presence of the sun in the FOV is detected, the miniDSS transitions into the tracking mode in which only a small regionof-interest (ROI) of $25 \times 25$ pixels around the coarse location is read-out to accurately determine the center of the sun spot. If sun presence is lost as result of the sun moving out of the FOV, the miniDSS transitions back into the acquisition mode.

\subsection{Acquisition mode}

The purpose of the acquisition mode is to determine the $25 \times 25$ pixel region-of-interest (ROI), which contains the complete sun spot of about 10 pixels in diameter. Conventionally, in acquisition mode the full pixel array is scanned pixel-by-pixel from top left to bottom right. However, in the miniDSS design this ROI is determined using a row and column profiling scheme. The profiling is achieved by a specific pixel design. The pixel array is surrounded by two addressing circuits: one for the $Y$ or row addressing, the other one for the $X$ or column addressing. By accessing all rows at once, the pixels on a single column are all simultaneously connected to the same read-out circuitry. The value that is read out is in that case dominated by the pixel with highest intensity within that column. I.e., if one of the pixels within that column is illuminated by the sun, the amplitude read out from that column will be high. Using this scheme, a profile of the columns with at least one pixel illuminated above a predefined threshold is rapidly obtained. A similar approach is used to find the rows comprising sun-illuminated pixels. Hence, the combination of rows and columns with highest amplitude specify the location of the ROI to use in the tracking mode. In order to avoid false sun spot detection as result of faulty, i.e. "always on" pixels, sun presence is only assumed if at least 3 adjacent columns and rows show amplitudes above the threshold. The row and column profiling is schematically depicted in Figure 3. Contrary to conventional digital sun sensors, as result of the employment of the profiling principle, acquisition and tracking modes are characterized by similar power consumption. Moreover, the miniDSS requires only the time to read-out two lines, one for row- and one for column-profiling, to determine the coarse sun position. Therefore, compared to conventional digital sun sensors, the miniDSS is characterized by a much shorter acquisition time.

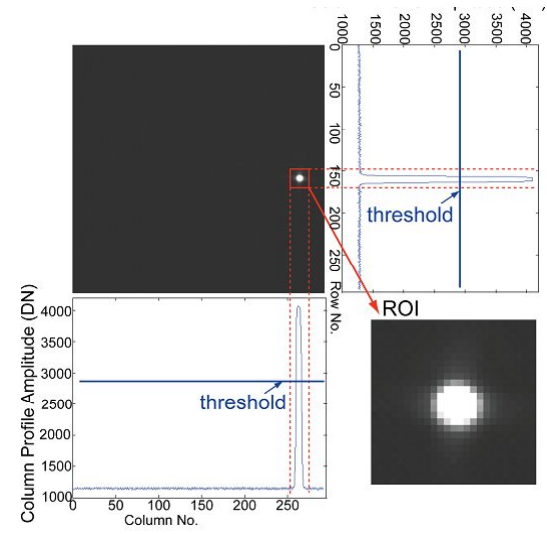

Figure 3: Schematic representation of the row and column profiling principle.

\subsection{Tracking mode}

Upon sun acquisition, the miniDSS transitions to the tracking mode in which only a window of $25 \times 25$ pixels, i.e. the ROI around the sun spot, commonly referred to as the tracking window, is read out. Using a proprietary centroiding algorithm, the center of the sun spot is accurately determined from the intensities registered by the pixels in the tracking window. The miniDSS periodically provides $X$ and $Y$ values with a precision of 15 bit to the S/C, representing the realtime sun-spot position. The first 9 bits identify the pixel closest to the sunspot center and the 6 remaining bits convey the deviation of the exact location of the sun spot center from that nearest pixel. Using this precision, a sub-pixel resolution of $1 / 64$ pixel is thus achieved.

Although in the vast majority of expected orbital positions the tracking window will not be illuminated by earth albedo, in limited situations part of the tracking window (ROI) may be illuminated, affecting the accuracy of the centroid algorithm somewhat. Analysis has shown that the maximum angular error as result of earth albedo in the worst case 
scenario can be expected to be $1.4 \cdot 10^{-3}{ }^{\circ}$. The miniDSS is therefore effectively albedo insensitive, which is considered a large advantage over analog sun sensors.

\section{DESIGN}

Figure 4 depicts a cross-section of the miniDSS design. The miniDSS is a effectively a single-chip sun sensor. The core of the miniDSS is the APS+ application-specific integrated circuit (ASIC). This chip is adhered to a ceramic chip carrier, which furthermore carries peripheral electronics and a ceramic spacer. This spacer supports the sapphire aperture, such that it remains at a well-known and stable distance above the pixel array on the APS+. The chip carrier is positioned in an aluminum housing of which the top-cover comprises a sapphire window, such that the sensor is exposed to direct sunlight.

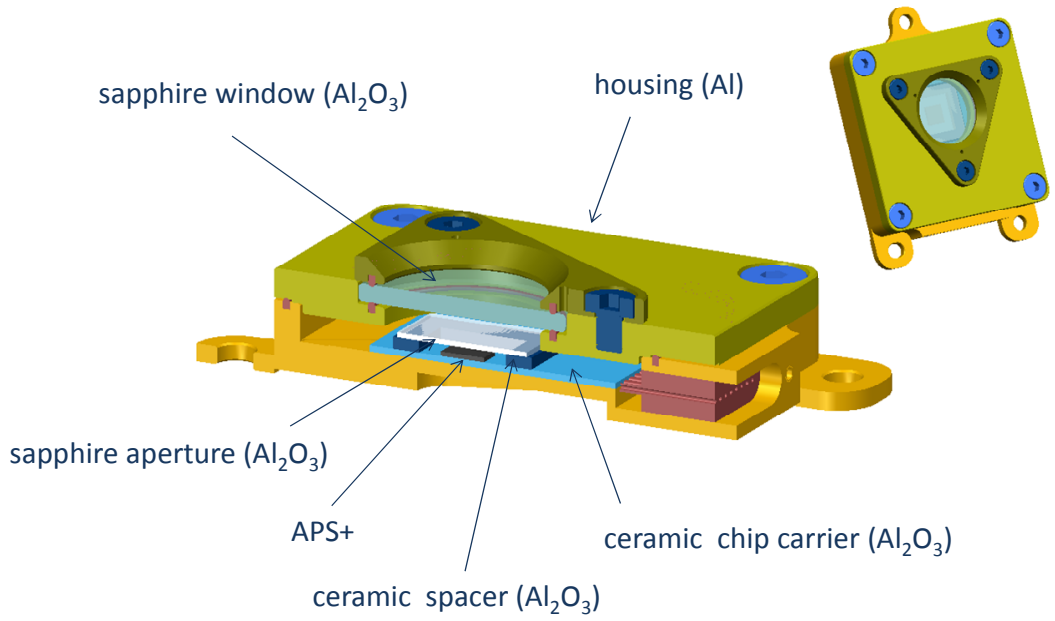

Figure 4: Cross-sectional view of the miniDSS design.

\subsection{APS+ ASIC}

The APS+ ASIC not only comprises a 2D active pixel array, but also the read-out circuitry, a sequencer, the sun centroid algorithm and communication circuitry. It was designed in a cost-effective 0.18 micron CMOS process and is characterized by low power consumption and high radiation tolerance. The APS+, which is shown in Figure 5, measures only $5 \times 5 \mathrm{~mm}^{2}$.
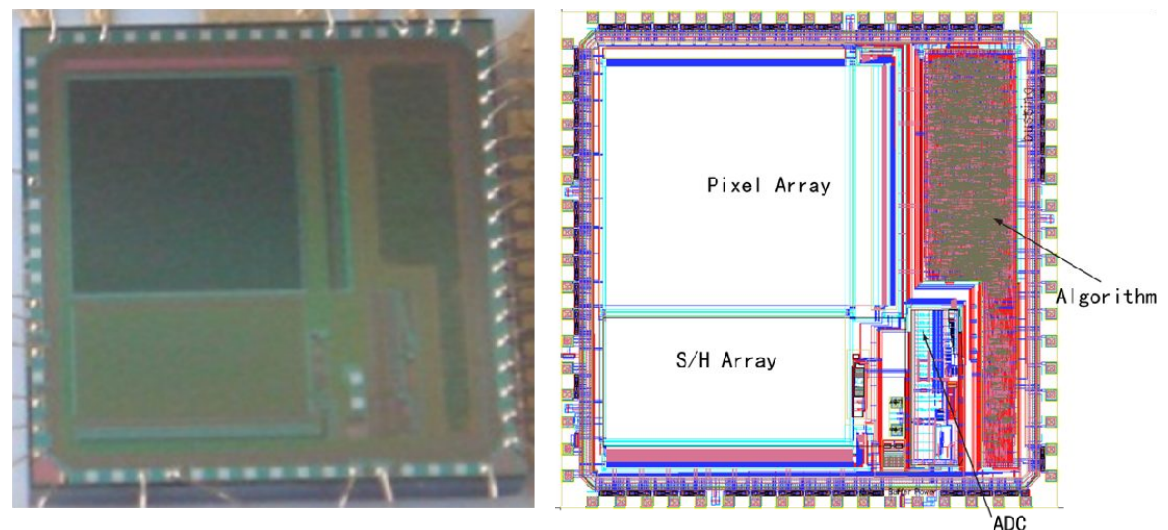

Figure 5: Photograph of the APS+ chip (left) and layout (right). 
The APS+ comprises $368 \times 368$ pixels having a pixel size of $6.5 \mu \mathrm{m}$. In order to minimize the probability of latch-up, the individual pixels comprise only p-type transistors. This design choice results in a lower quantum efficiency $(\sim 25 \%)$ compared to pixel arrays employing both p-type and n-type transistors. However, given the large intensity of the sun light, some quantum efficiency could easily be sacrificed, as to obtain a more radiation-tolerant sensor. Figure 6 shows the quantum efficiency of the APS + as a function of wavelength. To further reduce latch-up sensitivity, abundant substrate contacts have been implemented.

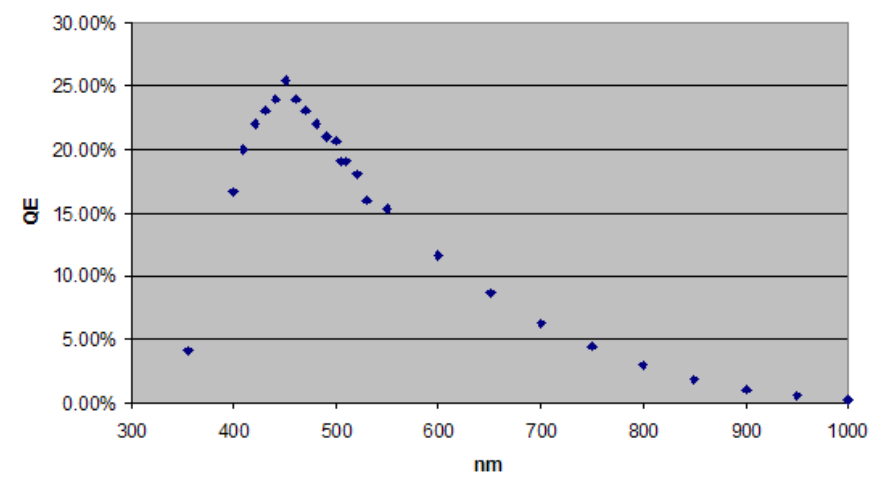

Figure 6: Quantum efficiency of the APS+ as a function of wavelength.

The read-out circuitry consists of drivers for the pixel array, as well as a 10-bit pipelined analog-to-digital converter (ADC) to covert the pixel signals to the digital domain. Switching between acquisition and tracking mode, control and timing is managed by the sequencer. The digital core furthermore comprises the centroiding algorithm, which determines the location of the sun spot, as well as the communication circuitry. Two mutually exclusive communication interfaces have been implemented: RS-422 (UART) and SPI.

Several settings can be changed by telecommand (TC). For example, the integration time can be changed individually for acquisition mode and tracking mode. Furthermore, the field of view can be reduced by reducing the active size of the array in steps of $1 / 4^{\text {th }}$ of the original size. This is particularly useful if part of the $\mathrm{S} / \mathrm{C}$, e.g. a solar array, is in the FOV of the miniDSS. In that case a smaller FOV can be chosen to prevent possible misidentification of the sun spot as result of direct reflection from the solar array.

\subsection{Housing}

The material used for the housing is aluminum. Its outer surfaces have been treated with alodine. Aluminum has a low density, is non-magnetic and easy to machine. Moreover, aluminum has a good thermal conductivity, which is advantageous from a thermal point of view. Since (small) S/C panels and brackets are typically made from aluminum as well, the miniDSS housing matches perfectly in term of thermal expansion. The alodine-treated surfaces of the package moreover provide a good interface for adhesion of the ceramic chip carrier and the integrated connector.

The optical interface of the housing consists of a $2 \mathrm{~mm}$ thick radiation tolerant sapphire window. The window is coated with an optical attenuation filter, which transmits less than $1 \%$ of the sun light, to match the pixel full well of the detector for useful exposure times, as to avoid saturation of the APS+ image sensor. Although sapphire can be welded into aluminum packages, it has been decided to clamp the window in the housing with conductive rubber seals on both sides, which are polymerized into the top-cover and triangular window clamp, respectively. This allows easy exchange of the optical window, e.g. for a window carrying a mission-specific attenuation filter. The conductive seal material, which is also used between the part of the housing that holds the chip carrier and the top-cover, is ECSS compliant with regard to total mass loss (TML) and Collected Volatile Condensable Materials (CVCM). An Indium Tin Oxide (ITO) coating on top of the window ensures electrical conductivity for all external surfaces. This prevents the accumulation of static charge on the exterior of the window.

An important feature for internal and external alignment of the sun sensor is the lay-out of the three mounting feet, which provide the mechanical interface of the miniDSS with the S/C. Figure 7 depicts the part of the housing comprising the mounting feet. They are co-planar and designed such that the right-bottom foot has a calliper hole, of which the center is the mechanical reference point for the miniDSS, the left-bottom foot has a slotted hole and the top foot has a slightly oversized hole. By applying specific fasteners with calliper shafts, rotational alignment of the sensor can be assured. 
These feet are also used during assembly of the APS+ chip and aperture to ensure accurate co-alignment of chip, aperture and housing.

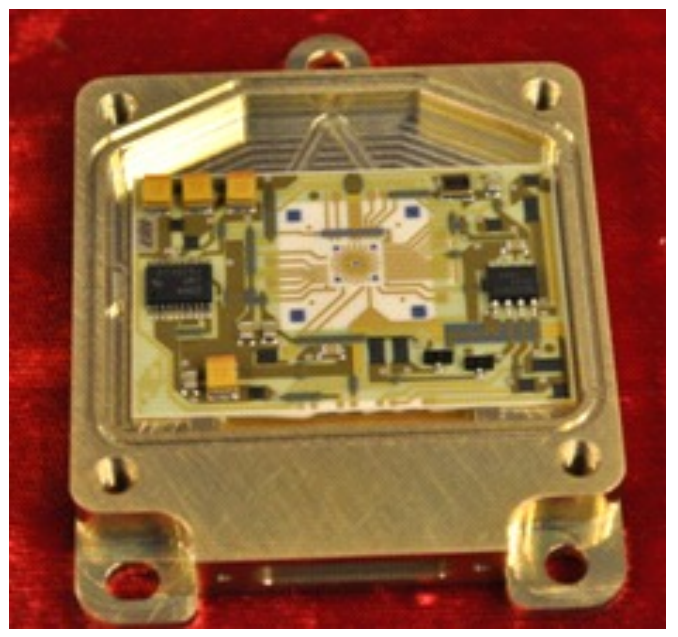

Figure 7: Housing comprising ceramic chip carrier carrying the SMD peripheral electronics.

\subsection{Chip carrier}

The ceramic chip carrier was produced by Lewicki GmbH (Germany). The chip carrier carries the bare APS+ chip, the surface mounted device (SMD) peripheral electronics, and the spacer with aperture. The chip carrier is adhered to the housing using a thermally conductive ceramic filled silicon adhesive. This adhesive is characterized by good performance regarding thermal cycling of bonded materials with different coefficients of thermal expansion (CTE), such as ceramic and aluminum. The distance between the APS+ and the aperture is controlled by a ceramic spacer, adhered to the chip carrier on one side and to the aperture on the other side. Figure 7 shows a photograph of the chip carrier with peripheral electronics in the housing, taken before assembly of the chip, spacer and aperture.

\subsection{Aperture}

The aperture consists of a metallization layer that is vacuum-deposited on the sapphire carrier. A pinhole with a diameter of $65 \mu \mathrm{m}$ is obtained in this metallization layer by employing a lift-off technique. Chip, spacer and aperture are assembled using pick and place machinery in order to minimize misalignment. The alignment is performed with reference to the mechanical reference frame. The use of pick and place technologies, allows for low-cost production of units with high uniformity.

The metallization layer also provides anti-reflex (AR) functionality. The proprietary coating was specifically designed to reduce the change of so-called ghost images. If no AR coating were to be used, sun light that is reflected from the APS+ IC surface could be reflected from the bottom side of the aperture back on to the pixel array. This could lead to misidentification of the sun spot location or a deterioration in the accuracy of the miniDSS. The latter could occur if part of the reflected light is incident on the tracking window. Figure 8 shows the simulated reflectance as a function of wavelength for the metallization layer at $0^{\circ}$ incidence. As can be seen in figure 7 , the reflectance is expected to be maximally $1 \%$ for the wavelength range where the quantum efficiency of the APS+ IC is highest.

For the sensor core, materials with well-matched CTE were selected, to maximize the number of thermal cycles that the unit is able to withstand. For example, both the chip carrier and the spacer that is used to maintain a well-controlled distance (collimator height $h$ ) between the pixel array surface and the aperture are ceramic $\left(\mathrm{Al}_{2} \mathrm{O}_{3}\right)$. Moreover, the aperture itself is made of radiation tolerant sapphire $\left(\mathrm{Al}_{2} \mathrm{O}_{3}\right)$. The coefficients of thermal expansion of these materials is in the order of $5.10^{-6} /{ }^{\circ} \mathrm{C}$, while that of the ASIC $(\mathrm{Si})$ is $3.10^{-6} /{ }^{\circ} \mathrm{C}$. 


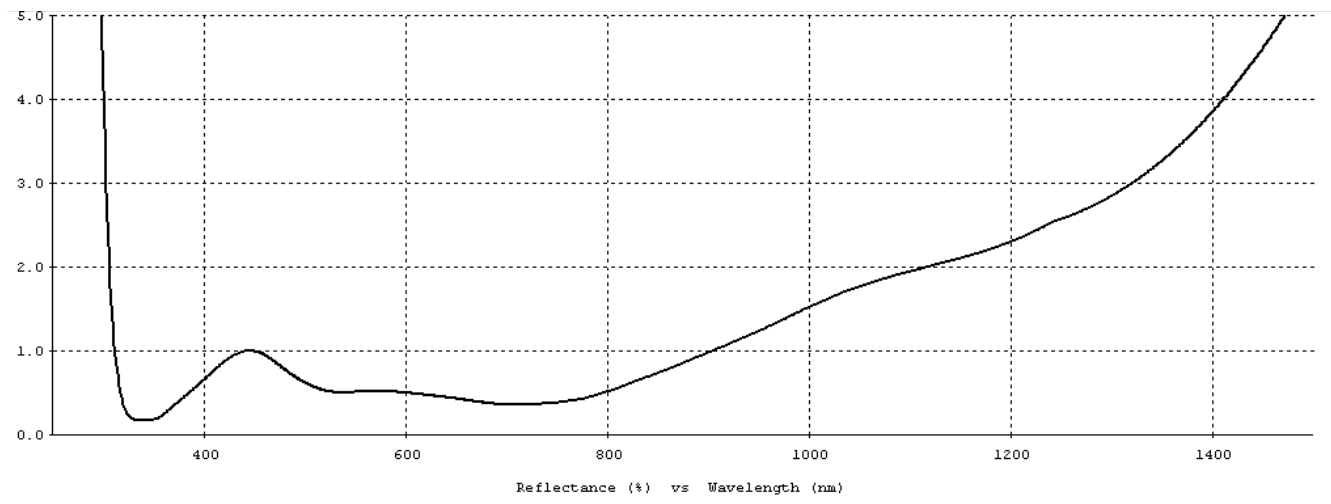

Figure 8: reflectance vs. wavelength for the AR coating at $0^{\circ}$ incidence.

\section{RESULTS}

The miniDSS prototype underwent a limited performance test, as well as a limited thermal vacuum (TVAC) test. In an initial calibration step, the misalignment of the aperture with respect to the APS+ chip was determined. In the $x$-direction the misalignment was found to be $-11 \mu \mathrm{m}$, while the misalignment in the $y$-direction was found to be $4.5 \mu \mathrm{m}$. These misalignment values fall within the expected manufacturing accuracy and were not notably affected by the TVAC test. The misalignments would result in a worst case sun aspect angle error of $0.28^{\circ}$, if not compensated for.

Aperture misalignment can in principle be determined based on sun aspect angle error measurements performed at just a few points in the FOV. The full calibration of a sun-sensor unit traditionally contributes significant to the recurrent unit cost. This is related to the complex stimulus that needs to be applied and the procedure in the cleanroom that takes about a one day of work for one sun sensor. Since the largest contribution to inaccuracy can be attributed to aperture misalignment, a mini-DSS calibration device is currently under development that would be capable to calibrate a miniDSS on the spot, even integrated on the S/C.

The resolution, noise equivalent angle (NEA), FOV and accuracy were measured using a sun simulator. The resolution was determined by scanning on the $\alpha$-axis with steps of $0.1^{\circ}$ between $-1^{\circ}$ and $1^{\circ}$. The resolution was obtained by dividing the $0.1^{\circ}$ angle step by the average change in $Y$-value as result of the $0.1^{\circ}$ steps and was found to be $0.007^{\circ}$. The accuracy is not limited by the resolution, i.e. other factors are dominant in causing the errors that limit the accuracy. At present, a higher resolution, e.g. obtained from employing a larger number of pixels or larger sub-pixel resolution, would not improve accuracy. The NEA was determined using an ensemble of measurements taken at 3 different locations in the FOV. The NEA was found to be $0.01^{\circ}(3 \sigma)$. The FOV was determined by scanning across both perpendicular axes consecutively with a resolution of $2^{\circ}$ until $50^{\circ}$ and with a resolution of $1^{\circ}$ from there on. The measured field of view is $102^{\circ} \times 102^{\circ}$. The field of view was found to be practically square, i.e. sun presence was for instance during a diagonal scan lost at $\alpha, \beta=49^{\circ}$, a mere $2^{\circ}$ less than when scanning on the $\alpha$ or $\beta$ axis. By changing the FOV parameter by telecommand, a FOV can be selected from $102^{\circ} \times 102^{\circ}, 91^{\circ} \times 91^{\circ}, 69^{\circ} \times 69^{\circ}$ and $38^{\circ} \times 38^{\circ}$.

The APS+ chip must be positioned at a well-known and stable distance behind the aperture. The distance between the chip and the aperture (i.e. collimator height $h$ ) is a key parameter in the conversion from pixel coordinates to sun aspect angles and should therefore be stable in time and with temperature to achieve a high sensor performance. In order to test, among others, the temperature stability of the collimator height, a TVAC test was performed. Figure 9 shows photographs of the sun simulator set-up at TNO that was used for the TVAC test and the miniDSS mounted in the simulator. The sun simulator uses a 1600W Xenon arc source in combination with a Balzers solar simulator filter (ST-87-2799/HB ) to remove the Xenon peaks as to better comply with the sun spectrum. Stray light baffles, and a Liesegang F3.6/330 lens are furthermore employed. This source produces a $89 \mathrm{~mm}$ diameter beam with an apparent sun diameter of about $1.5^{\circ}$. The integrated spectral intensity is approximately 1 solar constant AM0. The miniDSS is mounted on a vacuum rotation table RV240PP set-up (1 axis rotary unit). A Yokogawa temperature recorder with 3 PT-100 sensors; with an overall accuracy of $\pm 1^{\circ} \mathrm{C}$ was used to monitor the temperature of the miniDSS. 


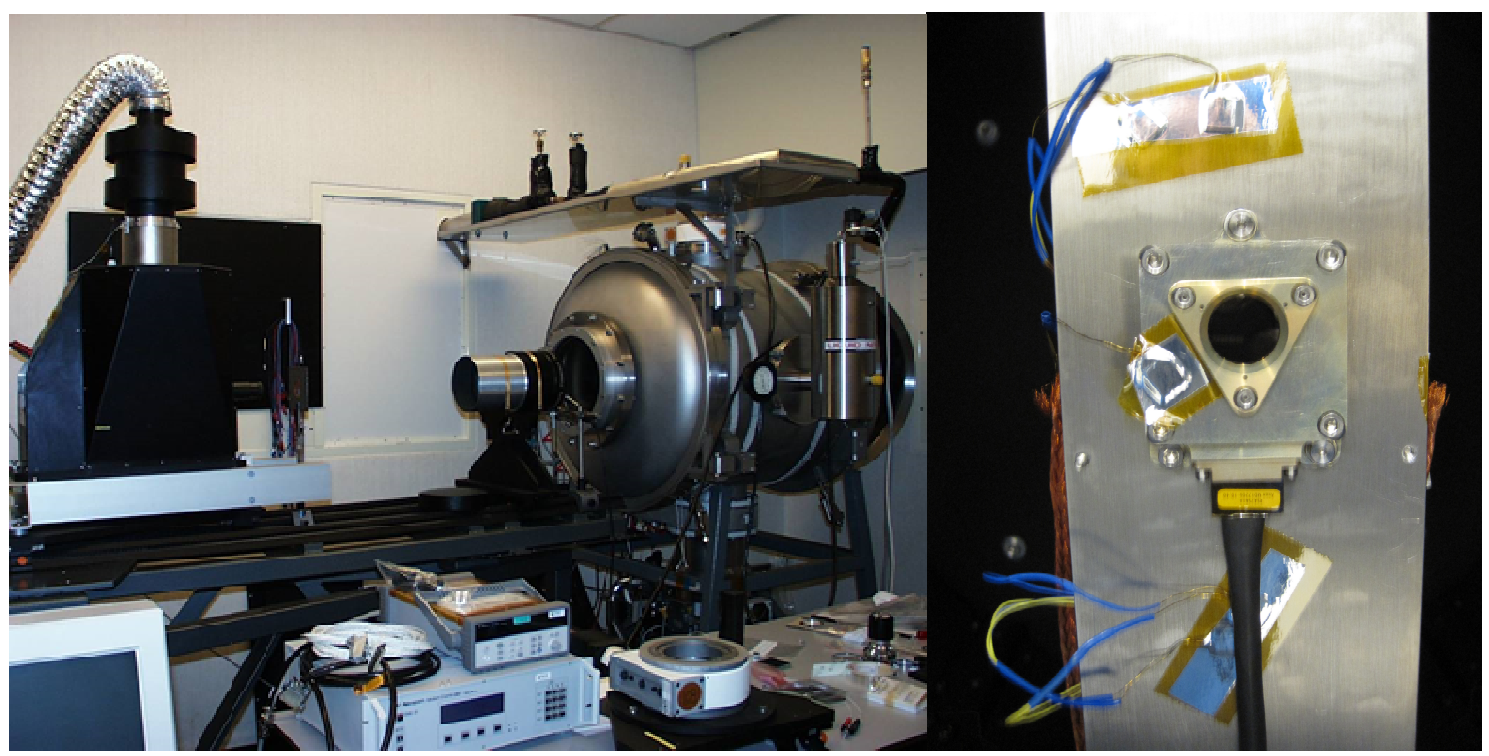

Figure 9: TNO sun simulator set-up (left) and miniDSS mounted in simulator (right)

The results of this test are shown in

Figure 10. This graph shows errors in sun aspect angle $\alpha$, i.e. the difference between the angle measured by the miniDSS and the angle at which light was incident on the miniDSS. The errors were measured during scans across the FOV in vacuum at three different temperatures: $-15^{\circ} \mathrm{C}, 25^{\circ} \mathrm{C}$ and $50^{\circ} \mathrm{C}$. As can be seen, the measured errors vary slightly with temperature and the temperature effect is largest at the edges of the FOV, as can be expected since the error is more sensitive to variations in the collimator height in those regions. However, overall the temperature effects are limited and never exceed roughly $0.02^{\circ}$ when reducing or increasing temperature from room temperature. Moreover, across the FOV and at all temperatures, the error never exceeds $0.03^{\circ}$, indicating that the accuracy, at least on the $\alpha$-axis, is about $0.03^{\circ}$. Further measurements are required to confirm that this accuracy is achieved across the complete FOV. It should be noted that the errors are rather systematic in nature, suggesting that an even higher accuracy could be achieved, albeit at increased calibration costs, if a full calibration LUT were to be used by the S/C.

It is believed that the rapid jumps in the measured errors around zero degrees angle, i.e. around the point where light is applied perpendicular to the miniDSS, is a remaining ghost image effect. Light reflected from the APS+ is reflected back from the bottom of the aperture and is incident on the tracking window, causing an error in the determination of sun spot position. As can be seen this effect does not significantly affect the overall accuracy of the miniDSS.

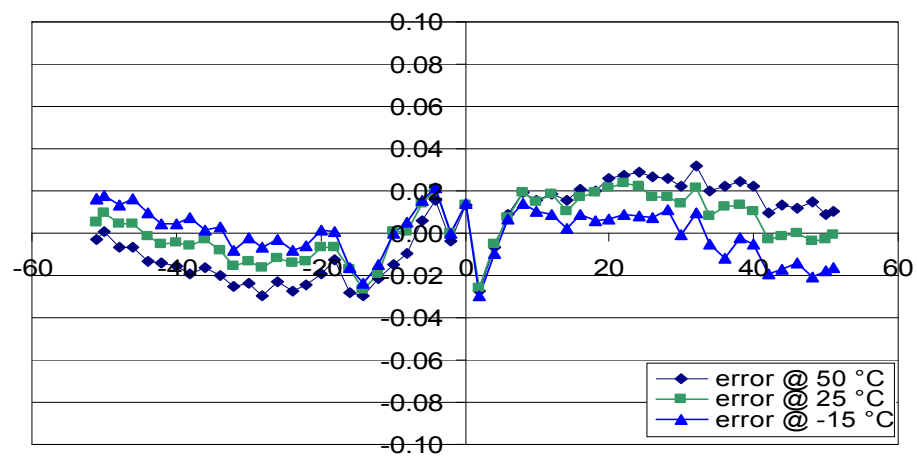

applied angle (deg)

Figure 10: Errors in sun aspect angle $\alpha$ measured during scans across the FOV in vacuum at different temperatures. 
This high accuracy combined with albedo insensitivity and low production cost, mass and power consumption suggest that the miniDSS could, in combination with other sensor units, also be used in modes of the attitude and orbital control systems (AOCS), other than safe mode or sun acquisition mode. For instance the sensor could also be used in modes where a moderately high pointing accuracy is required.

The miniDSS design was specifically optimized for low power consumption. The power consumption was measured to be only $65 \mathrm{~mW}$, which is roughly a factor 15 lower than found in conventional high-precision digital sun sensors ${ }^{1,2}$. Moreover, the power consumption was found to be practically independent of the operating mode. At dimensions of $69 \times 52 \times 14 \mathrm{~mm}^{3}$ and a mass of only 72 grams, the miniDSS has achieved an extreme reduction in both volume and weight as compared to the conventional devices, i.e. roughly a factor 10 and 5 , respectively.

\section{CONCLUSIONS}

A high-precision low-power miniaturized digital sun sensor has been developed at TNO. The miniDSS is a single-chip sun sensor comprising an ASIC with an APS detector. The prototype combines an accuracy in the order of $0.03^{\circ}$ with a mass of just $72 \mathrm{~g}$ and a power consumption of only $65 \mathrm{~mW}$. The sensor is effectively albedo insensitive and the design was optimized for low recurrent cost. This high accuracy combined with albedo insensitivity and low production cost, mass and power consumption suggest that the miniDSS could also be used in AOCS modes where a moderately high pointing accuracy is required. The miniDSS prototype is expected to be launched on QuadSat in 2013 for in-orbit demonstration.

\section{ACKNOWLEDGEMENTS}

The authors would like to gratefully acknowledge Bryan de Goeij for support during the performance analysis, Tom Duivenvoorde for the mechanical design of the housing, Jorge Fiebrich and Margreet Mol for performing AIT work and Andries Vis and Jacob-Jan van der Velde for their manufacturing contributions. The APS+ ASIC is the result of a fruitful collaboration between TNO and the Delft University of Technology. The authors would like to acknowledge H. Hakkesteegt and Henk Jansen from TNO, as well as A. Theuwissen and N. Xie from the Delft University of Technology for their efforts in the design of the APS+ ASIC. Further development towards qualified models is planned in close cooperation between TNO and Moog Bradford (formerly known as Bradford Engineering).

\section{REFERENCES}

[1] Boldrini, F., Monnini, E., Procopio, D., "Applications of APS detector to GNC sensors", Proc. 4th IAA Symposium on Small Satellites for Earth Observation, 2003

[2] de Boom, C.W., van der Heiden, N., "A novel digital sun sensor: development and qualification for flight", Proc. 54th International Astronautical Congress of the International Astronautical Federation (IAF), 2003. 